\title{
Printing of Cotton Fabrics Using Microwave Irradiation
}

\author{
Saminu Murtala Yakasai, Ismaila Shina Sadiq, \\ Amina Ahmad Lawal, Abdulkadir Ahmed Isah.
}

Department Of Chemistry, Federal University Dutse, Jigawa State.

Email:saminum@yahoo.co.uk

\begin{abstract}
Irradiation Microwave was used in fixation of pigment prints on cotton fabrics with a colour strength parameters comparable with the previous research obtained in case of thermo-fixation procedures. However printing pastes was introduced to the fabric via screen printing methods. The printed fabrics was subjected to irradiation microwave for different periods of time at microwave power. Results of both covered and uncovered ranges from 3.8 to 10.70 and 1.66 to 8.20.Also the effect of irradiation microwave on printed cotton fabrics with yellow pigment for $6 \mathrm{~min}$, for both covered and uncovered ranges from 3.50 to 15.52 and 5.20 to 16.80, likewise fastness properties of the cotton fabrics printed with yellow pigment fixed either by optimum conditions for irradiation microwave for both washing and rubbing which ranges from 4/5 for colour change(cc) and for colour staining(cs) which is 4 . In order to compare the initial result, another samples of cotton fabrics were printed with the same paste, dried and subjected to fixation procedure. The colour strength of the printed cotton fabrics fixed through irradiation microwave was found to be time and microwave power dependent. Results of this investigation clarified also that, regardless of the time of microwave, covering the pigment printed cotton fabric samples with covered and uncovered sample. Furthermore, the influence of reduction of the particle size of pigment yellow colour to the nano-scale on the colour strength and overall fastness properties of cotton fabrics printed and fixed using irradiation microwave was determined.
\end{abstract}

Keywords: Microwave, Irradiation, Screen, Fabric, Pigment.

\section{INTRODUCTION}

Printing involves the production of multiple copies of images or designs of various complexities and common with a range of colour. The printing process differs from the dyeing methods of colour application in that it involves the localized dyeing or pigmentation in a way dictated by the image. This localized application of color must be carried out with precision since each color of the image is applied with the help of a screen and registration of the print is paramount to the success of the operation. On an industrial scale, printing is used to provide repeat copies of artistic designs on textiles in a short period of time. However, the time taken depends upon the complexity of the design and the colorants, either dyes or pigments, used to color the image (freire, 2006).

However printing may have initiated in Egypt, India, or China at around 3000 BC. Wooden block printing centers existed in India around the first century and became prevalent in Europe in the fourteenth century. The use of stencils to make various coloured prints began to emerge from Japan in the seventeenth century. In 1752, Nixon initiated an engraved 
printing technique in Dublin. Copper roller printing became firmly recognized with several patents for the process being submitted by Bell in 1783. While 1850 the first silk-screen printing methods started to emerge in France. Rapid progress of this procedures followed and in 1976 screen printing had taken over from roller printing as the most important industrial process, utilizing both flat and rotary screens. Based on digital technology the ink jet process was introduced in 1990, and this technology soon began to impose a serious contest to previous printing techniques (Hertz and Samuelson, 1986).

Currently worldwide the production of printed textiles around the region of 20 billion meters. Although ink jet process has not superseded rotary screen printing in terms of machine running speed, ink jet lends itself to more complex, intricate designs and allows for shorter runs of cloth, making it a competitive process. There are also several other significant advantages of ink jet printing compared with either flat or rotary screen printing. For example, ink jet process does not require the costly operation of producing a screen for each colour to be printed, and there is less waste of printing paste (Soleimani-gorgani, 2015).

Meanwhile, process of textile printing, the insoluble pigment has affinity for the fiber, binding agents is used to fixed the pigment on to the textile material as the pattern required (Kipphan, 2001).

For several centuries, mineral pigments have been ground with natural binding agents such as drying and boiled oils, or viscous, aqueous solutions of albumen products and vegetable gums, and applied to the textile to form a pattern. The basic products for modern pigment printing were available quite early, but the technique required development (Gulrajani, 1993).

Infact, dispersion in 1920 is of useful organic pigments liquid were found in pigment printing today were available on the market. While around 1930 emulsion copolymerization of olefinic substances (such as vinyl esters, acrylonitrile, butadiene and acrylic acid esters was discovered at the former I G Forben in Leverkusen and Ludwigshafen (Jassal and Bajaj, 2001).

Pigment which is the largest colourant group for textile prints is of substantial importance since 1960. Majority of the textile prints in the modern world are printed using pigment printing, mainly because it is the simplest and the cheap printing procedure. The textile print met the market standards after drying and fixation, and washing process, carried out on chemical prints to remove unfixed dye, thickening agents and auxiliaries, is not normally when using the pigment printing technique (Clark, 1994).

In recent years, series of research were carried out searching for alternative source of energy to be clean, fast and attractive when it comes to textile printing and it was found that irradiation microwave is an efficient alternative source of energy in textile printing (Wynne, 1997).

Hence a lot of work and publication regarding the utilization of microwave in the field of textile dyeing has been carried out, but its usage in the textile printing is not yet achieved. However this work was carried out in view of evaluation of irradiation microwave to fix pigment printed textile fabrics, to determine the optimum printing conditions using microwave as source of energy (Haggag, 2014).

The print paste is the medium by which the colour that is pigment is applied to the textile substrate. Many print works will have their own distinctive formulations of print paste that Saminu M. Y. et al, DUJOPAS 7 (4): 60-67, 2021 
have been in used over series of years and in latter times were closely guarded secrets, known only to the master printer (Little and Christie , 2010).

In general, the formulation depends upon a number of factors, including the dye or pigments being printed, the substrate and the fastness requirements. The print paste must exhibit specific properties in order to achieve a successful print; the ingredients of the print paste and their functions are listed as follows (Kitchen et al., 2011):

1. Dye or pigment (provides color) dispersed)

2. Solvent (always water with textiles, the medium in which the other ingredients are dissolved).

3. Thickening agent (to increase the viscosity of the paste)

4. Solution assistants (to assist the dissolving of the ingredients)

5. Chemicals required to aid fixation of dyes (e.g. acids or alkalis)

6. Reducing or oxidizing agents (required by specific dyes for water solubilisation, also to prevent the reduction of dyes during steaming)

7. Humectants (assist in swelling the textile fiber during steaming)

The above list comprises the general ingredients of a generic print paste, and as stated earlier, the various print works will have variations on the above. Taking into consideration the colour, the print paste formulation is different when using a dye compared with a pigment and vice versa.

\section{Materials and Methods \\ Material}

The Bleached, Mill deseed and mercerized cotton fabrics produced by African textile manufacturers, for Spinning and Weaving, Kano, Nigeria (Substrate), While Commercial synthetic thickener Daicothick 1600 (Thickening Agent), Pigment used is commercial Pigment green under the name M. O. Green B while Binder used is commercial binder named Minex Binder BD.

Chemicals Reagents: Urea, sodium dihydrogen phosphate, dehydrate, binder, thickener, pigment and deionized water were of laboratory grade chemicals.

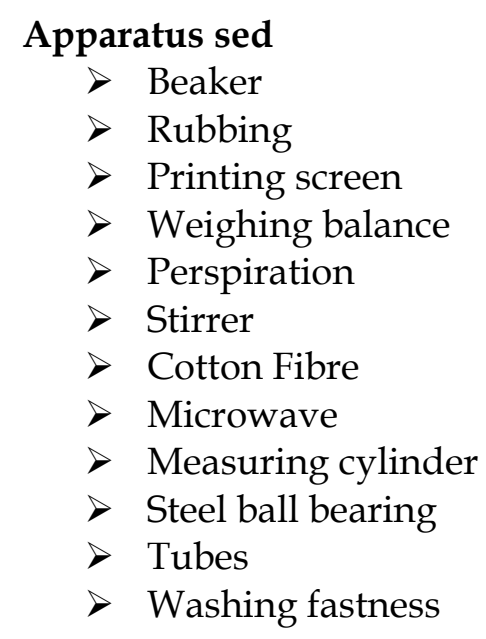

\section{Methods}

Preparation of Nano-scale Pigment: The particles of pigment were subjected to reduction using Ultrasonic homogenizer (Sonics and Materials, INC), Model: VCX750, Volts: 230VAC 50/60 HZNOM, U.S.A). 
Preparation of the Printing Paste

\begin{tabular}{|l|l|}
\hline Pigment Yellow & $8 \mathrm{~g}$ \\
\hline Urea & $5 \mathrm{~g}$ \\
\hline Pigment thickner & $8 \mathrm{~g}$ \\
\hline Binder & $10 \mathrm{~g}$ \\
\hline Sodium Dihydrogen Phosphate & $10 \mathrm{~g}$ \\
\hline Water & $\mathrm{Xg}$ \\
\hline Total & $200 \mathrm{~g}$ \\
\hline
\end{tabular}

The printing were applied to the fabric through screen printing process. After printing the printed fabric samples were subjected to drying to irradiation for different periods of time ranging between 2 to $10 \mathrm{mins}$ at different microwave power $(200,400,600,800$ and $1000 \mathrm{~W})$. in order to compare another samples of cotton fabrics were printed with the same paste, dried and subjected to fixation $\left(3 \mathrm{~min}\right.$ at $\left.140^{\circ} \mathrm{C}\right)$.

Washing

Immediately printing and fixation through irradiation, or thermo fixation the printed were subjected to washing through 5 stages as follows. Rinsing thoroughly with cold water, Treatment with hot water, treatment nears boiling temperate with a solution containing $2 \mathrm{~g} / 1$ sandpan, Washing with hot water, Rinsing with cold water. Finally the samples were dried and assessed for color strength (K/S).

Colour, Fastness Properties Measurements and Testing

The color strength of the printed samples was evaluated by Hunter lab Ultra scan PRO. The color strength, expressed as K/S and the overall fastness properties (washing, perspiration and crocking) were assessed according to the standard method.

\section{RESULTS}

Result

Table 1: Result of Time On Irradiation Microwave on Printed Cotton Fabrics With Yellow Pigment At $600 \mathrm{~W}$ Power.

\begin{tabular}{|c|c|c|}
\hline TIME/MIN & UNCOVERED SAMPLE & COVERED SAMPLE \\
\hline 2 & 1.66 & 3.80 \\
\hline 4 & 3.40 & 5.20 \\
\hline 6 & 4.80 & 7.60 \\
\hline 8 & 6.60 & 9.20 \\
\hline 10 & 8.20 & 10.70 \\
\hline
\end{tabular}

Colour Strength (K/S) Of The Sample Fix Via Conventional Method Thermo-Fixation=11.05

Table 2: Result of Power on Microwave Irradiation on Printed Cotton Fabrics With Yellow Pigment For 6 Min

\begin{tabular}{|c|c|c|}
\hline POWER(W) & UNCOVERED SAMPLE & COVERED SAMPLE \\
\hline 200 & 3.50 & 5.20 \\
\hline 400 & 6.00 & 7.80 \\
\hline 600 & 8.70 & 9.10 \\
\hline 800 & 13.47 & 14.10 \\
\hline 1000 & 15.52 & 16.80 \\
\hline
\end{tabular}

Colour Strenght (K/S) of The Sample Fix Via Conventional Method Thermo-Fixation=11.05 
Table 3- Fastness Properties of the Cotton Fabrics Printed With Yellow Pigment Fixed using Optimum Conditions For Irradiation.

\begin{tabular}{|c|c|c|c|c|c|c|c|c|}
\hline \multirow[t]{4}{*}{ Sample } & \multicolumn{8}{|c|}{ FastnessT Properties } \\
\hline & \multirow{2}{*}{\multicolumn{2}{|c|}{ Washing }} & \multirow{2}{*}{\multicolumn{2}{|c|}{ Rubbing }} & \multicolumn{4}{|c|}{ Perspiration } \\
\hline & & & & & \multicolumn{2}{|c|}{ Acidic } & \multicolumn{2}{|c|}{ Alkaline } \\
\hline & Cc. & Cs. & Wet. & Dry. & Cc. & Cs. & Cc. & Cs. \\
\hline $\begin{array}{l}\text { Pigment printed } \\
\text { fixed by irradiation } \\
\text { at } 1000 \mathrm{~W} \text { for } 6 \mathrm{~min}\end{array}$ & $4-5$ & 4 & $4-5$ & $4-5$ & $4-5$ & 4 & $4-5$ & 4 \\
\hline $\begin{array}{l}\text { Pigment printed by } \\
\text { non-irradiation. }\end{array}$ & $4-5$ & 4 & 4 & $4-5$ & $4-5$ & 4 & $4-5$ & 4 \\
\hline
\end{tabular}

Cc: Colour change, Cs: Colour staining

\section{Discussion}

A piece of material is introduced to irradiation microwave can be reflected from the surface if it is on electric conductor (graphite, metals etc), penetrate the material without absorption in the case of good insulators with good dielectric properties. (quartz glass, porcelain, and ceramics). But absorbed by the material if it is a lossy dielectric (i.e. material that exhibits socalled dielectric larses in turn results in heat generation in a quickly oscillating electromagnetic field such as water. In most cases, the electric field component of microwave radiation is responsible for electric heating mechanisms because it can cause molecular motion by either migration of ionic species (Wynne, 1997).

With this information, there are a lot of researches in the field of using microwave in textile dyeing; their usage in textile printing is not yet achieved.This may be due to the lack of water component in the printed film. The main aim of the present work is to evaluate the efficiency of $\mathrm{m}$ irradiation in fixation of pigment printing film. To achieve this goal a printing paste containing a yellow pigment colour was prepared according to the recipe indicated in the experimental section. The printed cotton fabric samples were subjected to microwave irradiation immediately after printing, i.e. before drying under a variety of conditions (Benford et al., 2015).

Effect of Different Irradiation Time and Differenrt Magnitudes Of Power: At different irradiation time at a constant microwave power at $600 \mathrm{~W}$ (table-1), or for $6 \mathrm{~min}$. at different magnitudes of power $(200,400,600,800$ and $1000 \mathrm{~W}$ (table-2). It is clear from the data of table- 1 that the K/S of the printed cotton fabrics fixed via microwave irradiation depends on the time of exposure, whereas the time of exposure increases from 2 to 4 to 6 to 8 and to 10 the K/S increases regularly from 1.66 to 3.40 to 4.80 to 6.60 to 8.20 respectively. It is also clear from table-1 that covering the pigment printed cotton fabric samples with either permeable cellulosic accompanied by a significant increase in K/S. This phenomenon holds true regardless of the time of microwave exposure, for example the sample which have been exposed for microwave irradiation at $600 \mathrm{~W}$ for $10 \mathrm{~min}$. increases from 8.20 to 10.70 by covering printed cotton fabric respectively(Kant, 2012).

Increasing the K/S by covering the printed goods is expected, since microwave heating depends on the polar solvent i.e. water in this study, which is able to absorb the electrical energy and convert it into heat energy. As the amount of water molecules on the printed surface of cotton fabric increases the expected heat on the fabric surface increases. Table- 2 
represent the values of K/S obtained when pigment printed cotton fabrics subjected to microwave fixation for $6 \mathrm{~min}$. at various magnitude of microwave power $(200,400,600,800$ and $1000 \mathrm{~W}$. it is clear from the data of table-2 that as the microwave power increases from 200 to $1000 \mathrm{~W}$ the K/S increases regularly from 3.50 to 15.52 in case of the uncover samples, from 5.20 to 16.80 and from for covered with respectively. Here, too covering the printed goods is followed by an increase in the K/S. In all cases the K/S follows the order uncover sample < covered sample (ATM)

From the results of table- 1 and table-2, it is clear that microwave could be used in fixation of pigment prints on cotton fabric samples. The K/S depends on both the power and time of microwave irradiation. A K/S of 8.20 and 15.52 could be achieved for the uncovered samples subjected to microwave irradiation for $10 \mathrm{~min}$. at $600 \mathrm{~W}$ or $6 \mathrm{~min}$. at $1000 \mathrm{~W}$ respectively while the $\mathrm{K} / \mathrm{S}$ of the sample printed by the same pigment colour and fixed via conventional technique, i.e. thermo-fixation at $140^{\circ} \mathrm{C}$ for $3 \mathrm{~min}$. acquire a $\mathrm{K} / \mathrm{S}$ of 11.05 (Polshettiwar and Varma, 2007)

Table-3 represents the overall colour fastness properties of the printed cotton fabric fixed either by irradiation. The data of table- 3 reveal that the overall colour fastness properties are nearly equal for both the samples either fixed via microwave irradiation or conventional methods (Kitchen et al., 2011).

Effect of Molecular Size of Pigment On the K/S and on the Overall Fastness Properties: Ultrafine pigments with excellent properties have widely been used in inkjet inks, dyeing of textile fibres and other fields. It was interested to investigate the effect of reduction of pigment particle to the non-irradiation methods on the quality of printing goods. Samples of cotton fabrics were printed using the two pastes and subjected to fixation at various powers $(800$ and $1000 \mathrm{~W})$ for different intervals of time (8 and 10min). For the sake of comparison another samples were printed and fixed via the conventional method,thermo-fixation at $140^{\circ} \mathrm{C}$ for 3 minutes, all the printed fabrics were washed and subjected to measure the $\mathrm{K} / \mathrm{S}$ and overall fastness properties.

Moreso these particle size plays a remarkable influence on the K/S of the printed goods. Finally, table-3 represent the overall colour fastness properties for cotton samples printed with conventional method or with yellowe pigment and fixed at the optimum condition, i.e. using microwave irradiation at $1000 \mathrm{~W}$ for 10 minutes. In fact c, from the data it is observed that decreasing the particle size of pigment yellow colour from 15.52 uncovered to 16.80 covered practically has no influence on the overall colour fastness properties (Latif et al., 2010).

\section{CONCLUSION}

It can be concluded that the irradiation could be used in pigment printing fixation on cotton fabric surface .It is also clear from the data that the K/S depends on: i. the size of particles, ii. the method of fixation, iii. The power and time of microwave fixation and iv. On the technique applied during microwave fixation, i.e. presence or absence of cover and also the nature of the cover. The values indicates the fastness properties where 4 means very good and 5 means excellent. Furthermore the data reveals that covering of the samples printed yellow pigment improves too the K/S. The K/S values of the pigment printing cotton fabric fixed by microwave irradiation follows the order uncovered sample $>$ covered sample. It is clear that the optimum conditions to achieve the higher $\mathrm{K} / \mathrm{S}$ on using nano-scale yellow pigment colour or conventional yellow pigment colour was at power of $1000 \mathrm{~W}$ for 10 minutes. The results obtained during the analysis were given below for both the covered Saminu M. Y. et al, DUJOPAS 7 (4): 60-67, 2021 
and uncovered which ranges from 3.8 to 0.70 and 1.66 to 8.20. Also for the effect of microwave irradiation on printed cotton fabrics with yellow pigment for $6 \mathrm{~min}$, for both covered and uncovered which ranges from 3.50 to 15.52 and 5.20 to 16.80, likewise for fastness properties of the cotton fabrics printed with yellow pigment fixed either by optimum conditions for microwave irradiation or conventional method for both washing and rubbing which ranges from $4 / 5$ for colour change(cc) and for colour staining(cs) which is 4 . For the sake of comparison another samples of cotton fabrics were printed with the same paste, dried and subjected to fixation according to the conventional technique. The $\mathrm{K} / \mathrm{S}$ values of the printed cotton fabrics fixed via microwave irradiation was found to be time and microwave power dependent. Results of this investigation clarified also that, regardless of the time of microwave, covering the pigment printed cotton fabric samples with covered and uncovered sample .Furthermore, the influence of minimization of the particle size of pigment yellow colour to the nano-scale on the K/S and overall fastness properties of cotton fabrics printed and fixed using microwave irradiation was also investigated.

\section{REFERENCES}

Clark, D.E.and W.H. (1994).Sutton, Microwave processing of materials, ANRMS, 26, 299-331.

Freire, M. (2006). Ink jet printing technology (CIJ/DOD) In: Ujiie, H. Digital printing of textiles. Woodhead

Gulrajani, M.L. (1993). Methods of Printing in Chemical Processing of Silk by Chopra,

Haggag K.A.(2014). Review Article of Microwave Irradiation and Its Application in Textile Industries, Science Publishing Group.

Hertz C.H. and Samuelsson B.A. (1986). Method and apparatus for high resolution ink jet printing. 4.620 .19

Kitchen H.J, Vallance, S.R, Kennedy, J.L, Tapia-Ruiz, N, Carassiti, L, Harrison, AG.(2011). Holme, Innovative technologies for high performance textiles, COLOR TECHNOL, 123, 5973 IJFTR,36, 58-62.

Benford J, Swegle, J.A, and Schamiloglu, E. (2015).High power microwaves, CRC Press;

Matharu, A.S. (2015). Green chemistry, biofuels, and biorefinery, Annu. Rev.

Jassal M. and Bajaj P., (2001).Developments in Acrylic Based Thickeners as Substitute of Emulsion Thickeners for Pigment Printing, Indian Journal of Fibre and Textile Research, 26(1-2), 143-155.

Kipphan, H. 2001. Handbook of print media, technologies and production methods. Springer, Berlin, Germany

Latif, A., Noor, S., Sharif, Q. M., \& Najeebullah, M. (2010). Different Techniques Recently Used For the Treatment of Textile Dyeing Effluents: A Review. Journal of the Chemical Society of Pakistan, 32(1), 115-124.

Little, A. F., \& Christie, R. M. (2010). Textile applications of photochromic dyes. Part 2: factors affecting the photocoloration of textiles screen-printed with commercial photochromic dyes. Coloration Technology, 126(3), 164-170. doi:10.1111/j.14784408.2010.00242.x

Kant R, Textile dyeing industry an environmental hazard, NSCI, 4, 22 (2012). A review on combustion synthesis intensification by means R.J. Giguere, Microwave-assisted organic synthesis, In MRS Proceedings, 269, 387. Cambridge.

Soleimani-Gorgani, A. 2015. Inkjet Printing. In: Izdebska, J. and Thomas, S., eds., Stemme, N. (1973). Printing on Polymers of writing mechanisms for writing on paper with a colored liquid. 374

Saminu M. Y. et al, DUJOPAS 7 (4): 60-67, 2021 
Polshettiwar V. , and R.S.Varma, R.S. (2007). Microwave-assisted organic synthesis and transformations Whittaker, TD.with a focus on ring-opening polymerization, Green Chem, 9,303-14 .

www.industrialmicrowave.com.

Wynne, A. (1997). Textiles. Macmillan Education Ltd., London. Improvement in Dyeability of Wool Fabric By Microwave Treatment, Y. Yan, Microwave-assisted synthesis of flower-like and leaf-like $\mathrm{CuO}$ nanostructures via room 